\title{
Generalized Fractional Integral Operators on Generalized Local Morrey Spaces
}

\author{
V. S. Guliyev, ${ }^{1,2}$ A. F. Ismayilova, ${ }^{2}$ A. Kucukaslan, ${ }^{3}$ and A. Serbetci ${ }^{3}$ \\ ${ }^{1}$ Department of Mathematics, Ahi Evran University, Bagbasi Campus, 40100 Kirsehir, Turkey \\ ${ }^{2}$ Institute of Mathematics and Mechanics, Academy of Sciences of Azerbaijan, B. Vahabzade Street 9, 1141 Baku, Azerbaijan \\ ${ }^{3}$ Department of Mathematics, Ankara University, Tandogan, 06100 Ankara, Turkey
}

Correspondence should be addressed to A. Serbetci; serbetci@ankara.edu.tr

Received 24 September 2014; Accepted 12 January 2015

Academic Editor: Igor E. Verbitsky

Copyright (C) 2015 V. S. Guliyev et al. This is an open access article distributed under the Creative Commons Attribution License, which permits unrestricted use, distribution, and reproduction in any medium, provided the original work is properly cited.

We study the continuity properties of the generalized fractional integral operator $I_{\rho}$ on the generalized local Morrey spaces $L M_{p, \varphi}^{\left\{x_{0}\right\}}$ and generalized Morrey spaces $M_{p, \varphi}$. We find conditions on the triple $\left(\varphi_{1}, \varphi_{2}, \rho\right)$ which ensure the Spanne-type boundedness of $I_{\rho}$ from one generalized local Morrey space $L M_{p, \varphi_{1}}^{\left\{x_{0}\right\}}$ to another $L M_{q, \varphi_{2}}^{\left\{x_{0}\right\}}, 1<p<q<\infty$, and from $L M_{1, \varphi_{1}}^{\left\{x_{0}\right\}}$ to the weak space $W L M_{q, \varphi_{2}}^{\left\{x_{0}\right\}}$, $1<q<\infty$. We also find conditions on the pair $(\varphi, \rho)$ which ensure the Adams-type boundedness of $I_{\rho}$ from $M_{p, \varphi^{1 / p}}$ to $M_{q, \varphi^{1 / q}}$ for $1<p<q<\infty$ and from $M_{1, \varphi}$ to $W M_{q, \varphi^{1 / q}}$ for $1<q<\infty$. In all cases the conditions for the boundedness of $I_{\rho}$ are given in terms of Zygmund-type integral inequalities on $\left(\varphi_{1}, \varphi_{2}, \rho\right)$ and $(\varphi, \rho)$, which do not assume any assumption on monotonicity of $\varphi_{1}(x, r)$, $\varphi_{2}(x, r)$, and $\varphi(x, r)$ in $r$.

\section{Introduction}

The theory of boundedness of classical operators of the real analysis, such as the maximal operator, Riesz potential, and the singular integral operators, from one weighted Lebesgue space to another one is well studied by now. Along with weighted Lebesgue spaces, Morrey-type spaces also play an important role in the theory of partial differential equations. Morrey spaces were first introduced by Morrey [1] in 1938 to study local behavior properties of the solutions of secondorder elliptic partial differential equations. Furthermore, there are important applications for the theory of partial differential equations related to obtaining sharp a priori estimates and studying regularity properties of solutions in Morrey spaces. Recently, they proved to be useful also for the Navier-Stokes equations [2,3]. However no attempt has been made to extend these results by using more generalized Morrey-type spaces. For example, sharp regularity properties of strong solutions to elliptic and parabolic equations with VMO coefficients in terms of general Morrey-type spaces are a good place to start the investigation.
For $x \in \mathbb{R}^{n}$ and $r>0$, we denote by $B(x, r)$ the open ball centered at $x$ of radius $r$ and by ${ }^{\complement} B(x, r)$ denote its complement. Let $|B(x, r)|$ be the Lebesgue measure of the ball $B(x, r)$.

Let $f \in L_{1}^{\text {loc }}\left(\mathbb{R}^{n}\right)$. The fractional maximal operator $M_{\alpha}$ and the Riesz potential $I_{\alpha}$ are defined by

$$
\begin{gathered}
M_{\alpha} f(x)=\sup _{t>0}|B(x, t)|^{-1+\alpha / n} \int_{B(x, t)}|f(y)| d y, \quad 0 \leq \alpha<n, \\
I_{\alpha} f(x)=\int_{\mathbb{R}^{n}} \frac{f(y) d y}{|x-y|^{n-\alpha}}, \quad 0<\alpha<n .
\end{gathered}
$$

If $\alpha=0$, then $M \equiv M_{0}$ is the Hardy-Littlewood maximal operator.

For a measurable function $\rho:(0, \infty) \rightarrow(0, \infty)$ the generalized Riesz potential $I_{\rho}$ is defined by

$$
I_{\rho} f(x)=\int_{\mathbb{R}^{n}} \frac{\rho(|x-y|)}{|x-y|^{n}} f(y) d y
$$


for any suitable function $f$ on $\mathbb{R}^{n}$. If $\rho(t) \equiv t^{\alpha}, 0<\alpha<n$, then we get the Riesz potential operator $I_{\alpha}$.

The generalized fractional integral operator $I_{\rho}$ was initially investigated in [4-6]. Nowadays many authors have been culminating important observations about $I_{\rho}$ especially in connection with Morrey spaces. Nakai [6] proved the boundedness of $I_{\rho}$ from the generalized Morrey spaces $M_{1, \varphi_{1}}$ to the spaces $M_{1, \varphi_{2}}$ for suitable functions $\varphi_{1}, \varphi_{2}$ satisfying the doubling condition. The boundedness of $I_{\rho}$ from the generalized Morrey spaces $M_{p, \varphi_{1}}$ to the spaces $M_{q, \varphi_{2}}$ is studied by Eridani [7], Gunawan [8], Eridani et al. [9], Nakai [10], and Eridani et al. [11]. Guliyev [12] proved the Spanneand Adams-type boundedness of $I_{\alpha}$ in the spaces $M_{p, \varphi}\left(\mathbb{R}^{n}\right)$ without any assumption on monotonicity of $\varphi$.

In this study, by using the method given by Guliyev in [13] (see also $[12,14]$ ), we prove the Spanne-type boundedness of the operator $I_{\rho}$ from one generalized local Morrey space $L M_{p, \varphi_{1}}^{\left\{x_{0}\right\}}$ to another one $L M_{q, \varphi_{2}}^{\left\{x_{0}\right\}}, 1<p<q<\infty$, and from $L M_{1, \varphi_{1}}^{\left\{x_{0}\right\}}$ to the weak space $W L M_{q, \varphi_{2}}^{\left\{x_{0}\right\}}, 1<q<\infty$. We also prove the Adams-type boundedness of the operator $I_{\rho}$ from generalized Morrey space $M_{p, \varphi^{1 / p}}$ to another one $M_{q, \varphi^{1 / q}}$ for $1<p<q<\infty$ and from $M_{1, \varphi}$ to $W M_{q, \varphi^{1 / q}}$ for $1<q<\infty$.

By $A \lesssim B$ we mean that $A \leq C B$ with some positive constant $C$ independent of appropriate quantities. If $A \leqslant B$ and $B \lesssim A$, we write $A \approx B$ and say that $A$ and $B$ are equivalent.

\section{Generalized Local Morrey Spaces $L M_{p, \varphi}^{\left\{x_{0}\right\}}$}

We find it convenient to define the generalized Morrey spaces in the form as follows.

Definition 1. Let $\varphi(x, r)$ be a positive measurable function on $\mathbb{R}^{n} \times(0, \infty)$ and $1 \leq p<\infty$. We denote by $M_{p, \varphi} \equiv M_{p, \varphi}\left(\mathbb{R}^{n}\right)$ the generalized Morrey space, the space of all functions $f \in$ $L_{p}^{\text {loc }}\left(\mathbb{R}^{n}\right)$ with finite quasinorm:

$$
\|f\|_{M_{p, \varphi}}=\sup _{x \in \mathbb{R}^{n}, r>0} \varphi(x, r)^{-1}|B(x, r)|^{-1 / p}\|f\|_{L_{p}(B(x, r))} .
$$

Also by $W M_{p, \varphi} \equiv W M_{p, \varphi}\left(\mathbb{R}^{n}\right)$ we denote the weak generalized Morrey space of all functions $f \in W L_{p}^{\text {loc }}\left(\mathbb{R}^{n}\right)$ for which

$$
\begin{aligned}
\|f\|_{W M_{p, \varphi}} & =\sup _{x \in \mathbb{R}^{n}, r>0} \varphi(x, r)^{-1}|B(x, r)|^{-1 / p}\|f\|_{W L_{p}(B(x, r))} \\
& <\infty
\end{aligned}
$$

According to this definition, we recover the Morrey space $M_{p, \lambda}$ and weak Morrey space $W M_{p, \lambda}$ under the choice $\varphi(x, r)=r^{(\lambda-n) / p}$ :

$$
\begin{aligned}
M_{p, \lambda} & =\left.M_{p, \varphi}\right|_{\varphi(x, r)=r^{(\lambda-n) / p}}, \\
W M_{p, \lambda} & =\left.W M_{p, \varphi}\right|_{\varphi(x, r)=r^{(\lambda-n) / p}} .
\end{aligned}
$$

Definition 2. Let $\varphi(x, r)$ be a positive measurable function on $\mathbb{R}^{n} \times(0, \infty)$ and $1 \leq p<\infty$. We denote by $L M_{p, \varphi} \equiv$
$L M_{p, \varphi}\left(\mathbb{R}^{n}\right)$ the generalized local (central) Morrey space, the space of all functions $f \in L_{p}^{\text {loc }}\left(\mathbb{R}^{n}\right)$ with finite quasinorm:

$$
\|f\|_{L M_{p, \varphi}}=\sup _{r>0} \varphi(0, r)^{-1}|B(0, r)|^{-1 / p}\|f\|_{L_{p}(B(0, r))} .
$$

Also by $W L M_{p, \varphi} \equiv W L M_{p, \varphi}\left(\mathbb{R}^{n}\right)$ we denote the weak generalized local (central) Morrey space of all functions $f \in$ $W L_{p}^{\text {loc }}\left(\mathbb{R}^{n}\right)$ for which

$$
\|f\|_{W L M_{p, \varphi}}=\sup _{r>0} \varphi(0, r)^{-1}|B(0, r)|^{-1 / p}\|f\|_{W L_{p}(B(0, r))}<\infty .
$$

Definition 3. Let $\varphi(x, r)$ be a positive measurable function on $\mathbb{R}^{n} \times(0, \infty)$ and $1 \leq p<\infty$. For any fixed $x_{0} \in \mathbb{R}^{n}$ we denote by $L M_{p, \varphi}^{\left\{x_{0}\right\}} \equiv L M_{p, \varphi}^{\left\{x_{0}\right\}}\left(\mathbb{R}^{n}\right)$ the generalized local Morrey space, the space of all functions $f \in L_{p}^{\text {loc }}\left(\mathbb{R}^{n}\right)$ with finite quasinorm:

$$
\|f\|_{L M_{p, \varphi}^{\left\{x_{0}\right\}}}=\left\|f\left(x_{0}+\cdot\right)\right\|_{L M_{p, \varphi}} .
$$

Also by $W L M_{p, \varphi}^{\left\{x_{0}\right\}} \equiv W L M_{p, \varphi}^{\left\{x_{0}\right\}}\left(\mathbb{R}^{n}\right)$ we denote the weak generalized local Morrey space of all functions $f \in W L_{p}^{\text {loc }}\left(\mathbb{R}^{n}\right)$ for which

$$
\|f\|_{W L M_{p, \varphi}^{\left\{x_{0}\right\}}}=\left\|f\left(x_{0}+\cdot\right)\right\|_{W L M_{p, \varphi}}<\infty .
$$

According to this definition, we recover the local Morrey space $L M_{p, \lambda}^{\left\{x_{0}\right\}}$ and weak local Morrey space $W L M_{p, \lambda}^{\left\{x_{0}\right\}}$ under the choice $\varphi\left(x_{0}, r\right)=r^{(\lambda-n) / p}$ :

$$
\begin{gathered}
L M_{p, \lambda}^{\left\{x_{0}\right\}}=\left.L M_{p, \varphi}^{\left\{x_{0}\right\}}\right|_{\varphi\left(x_{0}, r\right)=r^{(\lambda-n) / p}}, \\
W L M_{p, \lambda}^{\left\{x_{0}\right\}}=\left.W L M_{p, \varphi}^{\left\{x_{0}\right\}}\right|_{\varphi\left(x_{0}, r\right)=r^{(\lambda-n) / p}} .
\end{gathered}
$$

Furthermore, we have the following embeddings:

$$
\begin{array}{cl}
M_{p, \varphi} \subset L M_{p, \varphi}^{\left\{x_{0}\right\}}, & \|f\|_{L M_{p, \varphi}^{\left\{x_{0}\right\}}} \leq\|f\|_{M_{p, \varphi}}, \\
W M_{p, \varphi} \subset W L M_{p, \varphi}^{\left\{x_{0}\right\}}, & \|f\|_{W L M_{p, \varphi}^{\left\{x_{0}\right\}}} \leq\|f\|_{W M_{p, \varphi}} .
\end{array}
$$

Wiener $[15,16]$ looked for a way to describe the behavior of a function at infinity. The conditions he considered are related to appropriate weighted $L_{q}$ spaces. Beurling [17] extended this idea and defined a pair of dual Banach spaces $A_{q}$ and $B_{q^{\prime}}$, where $1 / q+1 / q^{\prime}=1$. To be precise, $A_{q}$ is a Banach algebra with respect to the convolution, expressed as a union of certain weighted $L_{q}$ spaces; the space $B_{q^{\prime}}$ is expressed as the intersection of the corresponding weighted $L_{q^{\prime}}$ spaces. Feichtinger [18] observed that the space $B_{q}$ can be described by

$$
\|f\|_{B_{q}}=\sup _{k \geq 0} 2^{-k n / q}\left\|f \chi_{k}\right\|_{L_{q}\left(\mathbb{R}^{n}\right)},
$$

where $\chi_{0}$ is the characteristic function of the unit ball $\{x \in$ $\left.\mathbb{R}^{n}:|x| \leq 1\right\}, \chi_{k}$ is the characteristic function of the annulus 
$\left\{x \in \mathbb{R}^{n}: 2^{k-1}<|x| \leq 2^{k}\right\}, k=1,2, \ldots$. By duality, the space $A_{q}\left(\mathbb{R}^{n}\right)$, called Beurling algebra now, can be described by

$$
\|f\|_{A_{q}}=\sum_{k=0}^{\infty} 2^{-k n / q^{\prime}}\left\|f \chi_{k}\right\|_{L_{q}\left(\mathbb{R}^{n}\right)} .
$$

Let $\dot{B}_{q}\left(\mathbb{R}^{n}\right)$ and $\dot{A}_{q}\left(\mathbb{R}^{n}\right)$ be the homogeneous versions of $B_{q}\left(\mathbb{R}^{n}\right)$ and $A_{q}\left(\mathbb{R}^{n}\right)$ by taking $k \in \mathbb{Z}$ in (42) and (13) instead of $k \geq 0$ there.

If $\lambda<0$ or $\lambda>n$, then $L M_{p, \lambda}^{\left\{x_{0}\right\}}\left(\mathbb{R}^{n}\right)=\Theta$, where $\Theta$ is the set of all functions equivalent to 0 on $\mathbb{R}^{n}$. Note that $L M_{p, 0}\left(\mathbb{R}^{n}\right)=$ $L_{p}\left(\mathbb{R}^{n}\right)$ and $L M_{p, n}\left(\mathbb{R}^{n}\right)=\dot{B}_{p}\left(\mathbb{R}^{n}\right)$ :

$$
\begin{array}{r}
\dot{B}_{p, \mu}=\left.L M_{p, \varphi}\right|_{\varphi(0, r)=r^{n+n p \mu}}, \\
W \dot{B}_{p, \mu}=\left.W L M_{p, \varphi}\right|_{\varphi(0, r)=r^{n+n p \mu}}, \\
\mu \in\left[-\frac{1}{p}, 0\right] .
\end{array}
$$

In order to study the relationship between central $B M O$ spaces and Morrey spaces, Alvárez et al. [19] introduced $\lambda$ central bounded mean oscillation spaces and central Morrey spaces $\dot{B}_{p, \mu}\left(\mathbb{R}^{n}\right) \equiv L M_{p, r^{n+n p \mu}}\left(\mathbb{R}^{n}\right), \mu \in[-1 / p, 0]$. If $\mu<-1 / p$ or $\mu>0$, then $\dot{B}_{p, \mu}\left(\mathbb{R}^{n}\right)=\Theta$. Note that $\dot{B}_{p,-1 / p}\left(\mathbb{R}^{n}\right)=L_{p}\left(\mathbb{R}^{n}\right)$ and $\dot{B}_{p, 0}\left(\mathbb{R}^{n}\right)=\dot{B}_{p}\left(\mathbb{R}^{n}\right)$. Also define the weak central Morrey spaces $W \dot{B}_{p, \mu}\left(\mathbb{R}^{n}\right) \equiv W L M_{p, n+n p \mu}\left(\mathbb{R}^{n}\right)$.

The classical result by Hardy-Littlewood-Sobolev states that if $1<p<q<\infty$, then the operator $I_{\alpha}$ is bounded from $L_{p}\left(\mathbb{R}^{n}\right)$ to $L_{q}\left(\mathbb{R}^{n}\right)$ if and only if $\alpha=n(1 / p-1 / q)$ and for $p=1<q<\infty$, the operator $I_{\alpha}$ is bounded from $L_{1}\left(\mathbb{R}^{n}\right)$ to $W L_{q}\left(\mathbb{R}^{n}\right)$ if and only if $\alpha=n(1-1 / q)$. Spanne and Adams studied boundedness of the Riesz potential in Morrey spaces. Their results can be summarized as follows.

Theorem 4 (Spanne, but published by Peetre [20]). Let $0<$ $\alpha<n, 1<p<n / \alpha$, and $0<\lambda<n-\alpha p$. Moreover, let $1 / p-1 / q=\alpha / n$ and $\lambda / p=\mu / q$. Then, for $p>1$, the operator $I_{\alpha}$ is bounded from $M_{p, \lambda}$ to $M_{q, \mu}$ and, for $p=1, I_{\alpha}$ is bounded from $M_{1, \lambda}$ to $W M_{q, \mu}$.

Theorem 5 (Adams [21]). Let $0<\alpha<n, 1<p<n / \alpha$, $0<\lambda<n-\alpha p$, and $1 / p-1 / q=\alpha /(n-\lambda)$. Then, for $p>1$, the operator $I_{\alpha}$ is bounded from $M_{p, \lambda}$ to $M_{q, \lambda}$ and, for $p=1$, $I_{\alpha}$ is bounded from $M_{1, \lambda}$ to $W M_{q, \lambda}$.

Some authors $[8,12,22-25]$ generalized Theorems 4 and 5 to generalized Morrey spaces and called them Spanne-type and Adams-type results for $I_{\alpha}$.

In [23] the following condition was imposed on $\varphi(x, r)$ :

$$
c^{-1} \varphi(x, r) \leq \varphi(x, t) \leq c \varphi(x, r)
$$

whenever $r \leq t \leq 2 r$, where $c \geq 1$ does not depend on $t, r$ and $x \in \mathbb{R}^{n}$, jointly with the condition

$$
\int_{r}^{\infty} t^{\alpha p} \varphi(x, t)^{p} \frac{d t}{t} \leq C r^{\alpha p} \varphi(x, r)^{p},
$$

where $C>0$ does not depend on $r$ and $x \in \mathbb{R}^{n}$.
In [23] the following Spanne-type result was proved for $I_{\alpha}$ on $M_{p, \varphi}$.

Theorem 6. Let $1<p<\infty, 0<\alpha<n / p, 1 / q=1 / p-$ $\alpha / n$, and $\varphi(x, r)$ satisfy the conditions (15) and (16). Then the operator $I_{\alpha}$ is bounded from $M_{p, \varphi}$ to $M_{q, \varphi}$.

The following Spanne-type result for $I_{\alpha}$ on $L M_{p, \varphi}^{\left\{x_{0}\right\}}$, containing results obtained in $[23,26]$, was proved in $[12,13]$ (see also [14]).

Theorem 7. Let $x_{0} \in \mathbb{R}^{n}, 1 \leq p<\infty, 0<\alpha<n / p, 1 / q=$ $1 / p-\alpha / n$, and $\left(\varphi_{1}, \varphi_{2}\right)$ satisfy the condition

$$
\int_{t}^{\infty} r^{\alpha} \varphi_{1}\left(x_{0}, r\right) \frac{d r}{r} \leq C \varphi_{2}\left(x_{0}, t\right),
$$

where $C$ does not depend on $x_{0}$ and $t$. Then the operator $I_{\alpha}$ is bounded from $L M_{p, \varphi_{1}}^{\left\{x_{0}\right\}}$ to $L M_{q, \varphi_{2}}^{\left\{x_{0}\right\}}$ for $p>1$ and from $L M_{1, \varphi_{1}}^{\left\{x_{0}\right\}}$ to $W L M_{q, \varphi_{2}}^{\left\{x_{0}\right\}}$ for $p=1$.

From Theorem 7 we get the following Spanne-type result for $I_{\alpha}$ on $M_{p, \varphi}$.

Corollary 8. Let $1 \leq p<\infty, 0<\alpha<n / p, 1 / q=1 / p-\alpha / n$, and $\left(\varphi_{1}, \varphi_{2}\right)$ satisfy the condition

$$
\int_{t}^{\infty} r^{\alpha} \varphi_{1}(x, r) \frac{d r}{r} \leq C \varphi_{2}(x, t),
$$

where $C$ does not depend on $x$ and $t$. Then the operator $I_{\alpha}$ is bounded from $M_{p, \varphi_{1}}$ to $M_{q, \varphi_{2}}$ for $p>1$ and from $M_{1, \varphi_{1}}$ to $W M_{q, \varphi_{2}}$ for $p=1$.

The following Spanne-type result for $I_{\alpha}$ on $M_{p, \varphi}$, containing results obtained in [12], was proved in [27].

Theorem 9. Let $1 \leq p<\infty, 0<\alpha<n / p, 1 / q=1 / p-\alpha / n$, and $\left(\varphi_{1}, \varphi_{2}\right)$ satisfy the condition

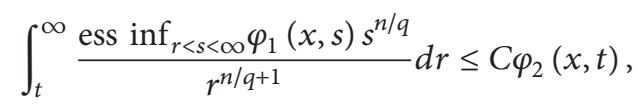

where $C$ does not depend on $x$ and $t$. Then the operator $I_{\alpha}$ is bounded from $M_{p, \varphi_{1}}$ to $M_{q, \varphi_{2}}$ for $1<p<q<\infty$ and from $M_{1, \varphi_{1}}$ to $W M_{q, \varphi_{2}}$ for $1<q<\infty$.

\section{Some Weighted Inequalities}

Let $v$ be a nonnegative function on $(0, \infty)$. We denote by $L_{\infty, v}(0, \infty)$ the space of all functions $g(t), t>0$, with finite norm

$$
\|g\|_{L_{\infty, \nu}(0, \infty)}=\sup _{t>0}(t)|g(t)|
$$

and $L_{\infty}(0, \infty) \equiv L_{\infty, 1}(0, \infty)$. Let $\mathfrak{M}(0, \infty)$ be the set of all Lebesgue-measurable functions on $(0, \infty)$ and $\mathfrak{M}^{+}(0, \infty)$ its subset consisting of all nonnegative functions on $(0, \infty)$. We 
denote by $\mathfrak{M}^{+}(0, \infty$; $\uparrow)$ the cone of all functions in $\mathfrak{M}^{+}(0, \infty)$ which are nondecreasing on $(0, \infty)$ and

$$
\mathbb{A}=\left\{\varphi \in \mathfrak{M}^{+}(0, \infty ; \uparrow): \lim _{t \rightarrow 0+} \varphi(t)=0\right\} .
$$

The following theorem is valid.

Theorem 10. Let $v_{1}, v_{2}$ be nonnegative measurable functions satisfying $0<\left\|v_{j}\right\|_{L_{\infty}(t, \infty)}<\infty, j=1,2$, for any $t>0$.

Then the identity operator $I$ is bounded from $L_{\infty, v_{1}}(0, \infty)$ to $L_{\infty, v_{2}}(0, \infty)$ on the cone $\mathbb{A}$ if and only if

$$
\left\|v_{2}\right\| v_{1}\left\|_{L_{\infty}(\cdot, \infty)}^{-1}\right\|_{L_{\infty}(0, \infty)}<\infty .
$$

Proof. If $F, G$ are nonnegative functions on $(0, \infty)$ and $F$ is nondecreasing, then

$$
\text { ess } \sup F(t) G(t)=\operatorname{ess} \sup F(t) \text { ess } \sup G(s), \quad t \in(0, \infty) \text {. }
$$

Also if $F, G$ are nonnegative functions on $(0, \infty)$ and $F$ is nonincreasing, then

$$
\underset{t \in(0, \infty)}{\operatorname{ess} \sup } F(t) G(t)=\underset{t \in(0, \infty)}{\operatorname{ess} \sup } F(t) \underset{s \in(0, t)}{\operatorname{ess} \sup } G(s), \quad t \in(0, \infty) .
$$

Therefore for all $\varphi \in \mathbb{A}$

$$
\text { ess } \sup v(t) \varphi(t)=\underset{t>0}{\operatorname{ess} \sup \varphi(t)} \bar{S} v(t)
$$

where

$$
(\bar{S} g)(t):=\|g\|_{L_{\infty}(t, \infty)}, \quad t \in(0, \infty) .
$$

First we prove sufficiency. Assume that condition (22) holds. Then for all $\varphi \in \mathbb{A}$

$$
\begin{aligned}
\|I \varphi\|_{L_{\infty, v_{2}}(0, \infty)} & =\left\|\left(\bar{S} v_{1}\right)^{-1} \bar{S} v_{1} \varphi\right\|_{L_{\infty, v_{2}}(0, \infty)} \\
& \leq \underset{t>0}{\operatorname{ess} \sup } \bar{S} v_{1}(t) \varphi(t) \cdot\left\|\left(\bar{S} v_{1}\right)^{-1}\right\|_{L_{\infty, v_{2}}(0, \infty)} \\
& =\left\|v_{2}\right\| v_{1}\left\|_{L_{\infty}(\cdot, \infty)}^{-1}\right\|_{L_{\infty}(0, \infty)} \operatorname{ess} \sup _{t>0}(t) \varphi(t)
\end{aligned}
$$

by (25).

To prove necessity assume that $I$ is bounded from $L_{\infty, v_{1}}(0, \infty)$ to $L_{\infty, v_{2}}(0, \infty)$ on the cone $\mathbb{A}$; that is,

$$
\|I \varphi\|_{L_{\infty, v_{2}}(0, \infty)} \leq c\|\varphi\|_{L_{\infty, v_{1}}(0, \infty)}, \quad \varphi \in \mathbb{A},
$$

where $c>0$ is independent of $\varphi$.

We note that $\left(\bar{S} v_{1}\right)^{-1} \chi_{(1 / n, \infty)} \in \mathbb{A}$ for all $n \in \mathbb{N}$ and take $\varphi=\left(\bar{S} v_{1}\right)^{-1} \chi_{(1 / n, \infty)}$. Observe that

$$
\begin{aligned}
& \left\|\left(\bar{S} v_{1}\right)^{-1} \chi_{(1 / n, \infty)}\right\|_{L_{\infty, v_{1}}(0, \infty)} \leq\left\|\left(\bar{S} v_{1}\right)^{-1}\right\|_{L_{\infty, v_{1}}(0, \infty)}
\end{aligned}
$$

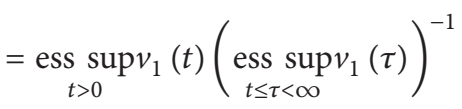$$
\leq 1 \text {. }
$$

Also for all $\tau>0$ for sufficiently large $n$

$$
\begin{aligned}
& \left\|\left(\bar{S} v_{1}\right)^{-1} \chi_{(1 / n, \infty)}\right\|_{L_{\infty, v_{2}}(0, \infty)} \\
& \geq\left\|\operatorname{ess}_{t \leq s<\infty} \chi_{(1 / n, \infty)}(s)\left(\bar{S} v_{1}\right)^{-1}(s)\right\|_{L_{\infty, v_{2}}(\tau, \infty)} \\
& =\left\|\left(\bar{S}_{1}\right)^{-1} v_{2}\right\|_{L_{\infty}(\tau, \infty)} ;
\end{aligned}
$$

hence

$$
\left\|\left(\bar{S}_{1}\right)^{-1} v_{2}\right\|_{L_{\infty}(\tau, \infty)} \leq c
$$

for all $\tau>0$ and condition (22) follows.

We will use the following statement on the boundedness of the weighted Hardy operator:

$$
H_{w} g(t):=\int_{t}^{\infty} g(s) w(s) d s, \quad 0<t<\infty,
$$

where $w$ is a weight.

The following theorem was proved in [28] (see, also [29]).

Theorem 11. Let $v_{1}, v_{2}$, and $w$ be weights on $(0, \infty)$ and let $v_{1}(t)$ be bounded outside a neighborhood of the origin. The inequality

$$
\underset{t>0}{\text { ess } \sup } v_{2}(t) H_{w} g(t) \leq \text { Cess } \sup _{t>0} v_{1}(t) g(t)
$$

holds for some $C>0$ for all nonnegative and nondecreasing $g$ on $(0, \infty)$ if and only if

$$
B:=\sup _{t>0} v_{2}(t) \int_{t}^{\infty} \frac{w(s) d s}{\operatorname{ess} \sup _{s<\tau<\infty} v_{1}(\tau)}<\infty .
$$

Moreover, the value $C=B$ is the best constant for (33).

Remark 12. In (33) and (34) it is assumed that $1 / \infty=0$ and $0 \cdot \infty=0$.

\section{Spanne-Type Result for the Operator $I_{\rho}$ in $L M_{p, \varphi}^{\left\{x_{0}\right\}}$}

We assume that

$$
\int_{1}^{\infty} \frac{\rho(t)}{t^{n}} \frac{d t}{t}<\infty
$$

so that the fractional integrals $I_{\rho} f$ are well defined, at least for characteristic functions $1 /|x|^{2 n}$ of complementary balls:

$$
f(x)=\frac{\chi_{\mathbb{R}^{n} \backslash B(0,1)}(x)}{|x|^{2 n}} .
$$

In addition, we will also assume that $\rho$ satisfies the growth condition: there exist constants $C_{1}>0$ and $0<2 k_{1}<k_{2}<\infty$ such that

$$
\sup _{r / 2<s \leq 3 r / 2} \frac{\rho(s)}{s^{n}} \leq C_{1} \int_{k_{1} r}^{k_{2} r} \frac{\rho(t)}{t^{n}} \frac{d t}{t}, \quad r>0 .
$$


This condition is weaker than the usual doubling condition for the function $\rho(t) / t^{n}$ : there exists a constant $C_{2}>0$ such that

$$
\frac{1}{C_{2}} \frac{\rho(t)}{t^{n}} \leq \frac{\rho(r)}{r^{n}} \leq C_{2} \frac{\rho(t)}{t^{n}},
$$

whenever $r$ and $t$ satisfy $r, t>0$ and $1 / 2 \leq r / t \leq 2$.

In the sequel for the generalized fractional integral operator $I_{\rho}$ we always assume that $\rho$ satisfies the conditions (37) and then denote the set of all such functions by $\widetilde{G}_{0}$. We will write, when $\rho \in \widetilde{G}_{0}$,

$$
\widetilde{\rho}(r):=C r^{n} \int_{r}^{\infty} \frac{\rho(t)}{t^{n}} \frac{d t}{t} .
$$

Remark 13. Typical examples of $\rho(t)$ that we envisage are, for $0<\alpha<n$,

$$
\rho(t) \equiv \begin{cases}t^{\alpha} \log \left(\frac{e}{t}\right), & 0<t \leq 1, \\ \frac{t^{\alpha}}{\log (e t)}, & 1 \leq t<\infty\end{cases}
$$

and, for $c>0$,

$$
\rho(t) \equiv \begin{cases}t^{\alpha}, & 0<t \leq 1 \\ e^{c} e^{-c t^{2}}, & 1 \leq t<\infty\end{cases}
$$

The second one is used to control the Bessel potential (see also [30]).

The following theorem was proved in [11].

Theorem 14. (1) Let $1<p<q<\infty$. Then the operator $I_{\rho}$ is bounded from $L_{p}\left(\mathbb{R}^{n}\right)$ to $L_{q}\left(\mathbb{R}^{n}\right)$ if and only if there exists $C>0$ such that for all $r>0$

$$
\rho(r) \leq C r^{n / p-n / q} .
$$

(2) Let $1<q<\infty$. Then the operator $I_{\rho}$ is bounded from $L_{1}\left(\mathbb{R}^{n}\right)$ to $W L_{q}\left(\mathbb{R}^{n}\right)$ if and only if there exists $C>0$ such that for all $r>0$

$$
\rho(r) \leq C r^{n-n / q} \text {. }
$$

The following lemma is valid.

Lemma 15. Let $1 \leq p<\infty$ and $\rho(t)$ satisfy the conditions (35) and (37). If the condition (42) is fulfilled, then for $p>1$ the inequality

$$
\begin{aligned}
\left\|I_{\rho} f\right\|_{L_{q}\left(B\left(x_{0}, r\right)\right)} \lesssim & \|f\|_{L_{p}\left(B\left(x_{0}, 2 r\right)\right)} \\
& +r^{n / q} \int_{2 r}^{\infty}\|f\|_{L_{p}\left(B\left(x_{0}, t\right)\right)} \frac{\rho(t)}{t^{n / p}} \frac{d t}{t}
\end{aligned}
$$

holds for any ball $B\left(x_{0}, r\right)$ and for all $f \in L_{p}^{\text {loc }}\left(\mathbb{R}^{n}\right)$.

If the condition (43) is fulfilled, then for $p=1$ the inequality

$\left\|I_{\rho} f\right\|_{W L_{q}\left(B\left(x_{0}, r\right)\right)} \leqslant\|f\|_{L_{1}\left(B\left(x_{0}, 2 r\right)\right)}$

$$
+r^{n / q} \int_{2 r}^{\infty}\|f\|_{L_{1}\left(B\left(x_{0}, t\right)\right)} \frac{\rho(t)}{t^{n}} \frac{d t}{t}
$$

holds for any ball $B\left(x_{0}, r\right)$ and for all $f \in L_{1}^{\text {loc }}\left(\mathbb{R}^{n}\right)$.
Proof. Let $1<p<\infty, 0<\alpha<n / p$, and $1 / q=1 / p-\alpha / n$. For arbitrary $x_{0} \in \mathbb{R}^{n}$, set $B=B\left(x_{0}, r\right)$ for the ball centered at $x_{0}$ and of radius $r$. Write $f=f_{1}+f_{2}$ with $f_{1}=f \chi_{2 B}$ and $f_{2}=f \chi_{c_{(2 B)}}$. Hence

$$
\left\|I_{\rho} f\right\|_{L_{q}(B)} \leq\left\|I_{\rho} f_{1}\right\|_{L_{q}(B)}+\left\|I_{\rho} f_{2}\right\|_{L_{q}(B)} .
$$

Since $f_{1} \in L_{p}\left(\mathbb{R}^{n}\right), I_{\rho} f_{1} \in L_{q}\left(\mathbb{R}^{n}\right)$ and from condition (42) we get the boundedness of $I_{\rho}$ from $L_{p}\left(\mathbb{R}^{n}\right)$ to $L_{q}\left(\mathbb{R}^{n}\right)$ (see Theorem 14) and it follows that

$$
\left\|I_{\rho} f_{1}\right\|_{L_{q}(B)} \leq\left\|I_{\rho} f_{1}\right\|_{L_{q}\left(\mathbb{R}^{n}\right)} \leq C\left\|f_{1}\right\|_{L_{p}\left(\mathbb{R}^{n}\right)}=C\|f\|_{L_{p}(2 B)},
$$

where constant $C>0$ is independent of $f$.

It is clear that $x \in B, y \in{ }^{C}(2 B)$ implies $(1 / 2)\left|x_{0}-y\right| \leq$ $|x-y| \leq(3 / 2)\left|x_{0}-y\right|$. Then from conditions (35), (37) and by Fubini's theorem we have

$$
\begin{aligned}
& \int_{\mathcal{C}_{(2 B)}} \frac{\rho(|x-y|)}{|x-y|^{n}}|f(y)| d y \\
& \leq \int_{C_{(2 B)}}|f(y)|\left(\int_{k_{1}\left|x_{0}-y\right|}^{k_{2}\left|x_{0}-y\right|} \frac{\rho(t)}{t^{n+1}} d t\right) d y \\
& \approx \int_{2 k_{1} r}^{\infty}\left(\int_{2 k_{1} r<\left|x_{0}-y\right|<t}|f(y)| d y\right) \frac{\rho(t)}{t^{n+1}} d t \\
& \lesssim \int_{2 r}^{\infty}\left(\int_{B\left(x_{0}, t\right)}|f(y)| d y\right) \frac{\rho(t)}{t^{n+1}} d t .
\end{aligned}
$$

Applying Hölder's inequality, we get

$$
\int_{\mathcal{C}_{(2 B)}} \frac{\rho(|x-y|)}{|x-y|^{n}}|f(y)| d y \leqslant \int_{2 r}^{\infty}\|f\|_{L_{p}\left(B\left(x_{0}, t\right)\right)} \frac{\rho(t)}{t^{n / p+1}} d t .
$$

Moreover, for all $p \in[1, \infty)$, the inequality

$$
\left\|I_{\rho} f_{2}\right\|_{L_{q}(B)} \leqslant r^{n / q} \int_{2 r}^{\infty}\|f\|_{L_{p}\left(B\left(x_{0}, t\right)\right)} \frac{\rho(t)}{t^{n / p+1}} d t
$$

is valid. Thus

$$
\left\|I_{\rho} f\right\|_{L_{q}(B)} \leqslant\|f\|_{L_{p}(2 B)}+r^{n / q} \int_{2 r}^{\infty}\|f\|_{L_{p}\left(B\left(x_{0}, t\right)\right)} \frac{\rho(t)}{t^{n / p+1}} d t .
$$

Let $p=1$. From the weak $(1, q)$ boundedness of $I_{\rho}$ and (43) it follows that

$$
\left\|I_{\rho} f_{1}\right\|_{W L_{q}(B)} \leq\left\|I_{\rho} f_{1}\right\|_{W L_{q}\left(\mathbb{R}^{n}\right)} \leqslant\left\|f_{1}\right\|_{L_{1}\left(\mathbb{R}^{n}\right)}=\|f\|_{L_{1}(2 B)} .
$$

Then from (50) and (52) we get the inequality (45).

The following theorem is one of the main results of this paper. 
Theorem 16. Let $x_{0} \in \mathbb{R}^{n}, 1 \leq p<\infty$, and the function $\rho$ satisfy the conditions (35), (37), and (42). Let also $\left(\varphi_{1}, \varphi_{2}\right)$ satisfy the conditions

$$
\begin{gathered}
\underset{t<s<\infty}{\operatorname{essinf}} \varphi_{1}\left(x_{0}, s\right) s^{n / p} \leq C \varphi_{2}\left(x_{0}, \frac{t}{2}\right) t^{n / q}, \\
\int_{r}^{\infty}\left(\underset{t<s<\infty}{\operatorname{essinf}} \varphi_{1}\left(x_{0}, s\right) s^{n / p}\right) \frac{\rho(t)}{t^{n / p+1}} d t \leq C \varphi_{2}\left(x_{0}, r\right),
\end{gathered}
$$

where $C$ does not depend on $x_{0}$ and $r$. Then the operator $I_{\rho}$ is bounded from $L M_{p, \varphi_{1}}^{\left\{x_{0}\right\}}$ to $L M_{q, \varphi_{2}}^{\left\{x_{0}\right\}}$ for $p>1$ and from $L M_{1, \varphi_{1}}^{\left\{x_{0}\right\}}$ to $W L M_{q, \varphi_{2}}^{\left\{x_{0}\right\}}$ for $p=1$. Moreover, for $p>1$,

$$
\left\|I_{\rho} f\right\|_{L M_{q, \varphi_{2}}^{\left\{x_{0}\right\}}} \leqslant\|f\|_{L M_{p, \varphi_{1}}^{\left\{x_{0}\right\}}},
$$

and, for $p=1$,

$$
\left\|I_{\rho} f\right\|_{W L M_{q, \varphi_{2}}^{\left\{x_{0}\right\}}} \leqslant\|f\|_{L M_{1, \varphi_{1}}^{\left\{x_{0}\right\}}}
$$

Proof. By Lemma 15 and Theorems 10 and 11 we have, for $p>$ 1 ,

$$
\begin{aligned}
\left\|I_{\rho} f\right\|_{L M_{q, \varphi_{2}}^{\left\{x_{0}\right\}}} \lesssim & \sup _{r>0} \varphi_{2}\left(x_{0}, r\right)^{-1} r^{-n / q}\|f\|_{L_{p}\left(B\left(x_{0}, 2 r\right)\right)} \\
& +\sup _{r>0} \varphi_{2}\left(x_{0}, r\right)^{-1} \int_{r}^{\infty}\|f\|_{L_{p}\left(B\left(x_{0}, t\right)\right)} \frac{\rho(t)}{t^{n / p+1}} d t \\
\approx & \sup _{r>0} \varphi_{1}\left(x_{0}, r\right)^{-1} r^{-n / p}\|f\|_{L_{p}\left(B\left(x_{0}, r\right)\right)} \\
= & \|f\|_{L M_{p, \varphi_{1}}^{\left\{x_{0}\right\}},}
\end{aligned}
$$

and, for $p=1$,

$$
\begin{aligned}
& \left\|I_{\rho} f\right\|_{W L M_{q_{,} \varphi_{2}}^{\left\{x_{0}\right\}}} \lesssim \sup _{r>0} \varphi_{2}\left(x_{0}, r\right)^{-1} r^{-n / q}\|f\|_{L_{1}\left(B\left(x_{0}, 2 r\right)\right)} \\
& +\sup _{r>0} \varphi_{2}\left(x_{0}, r\right)^{-1} \int_{r}^{\infty}\|f\|_{L_{1}\left(B\left(x_{0}, t\right)\right)} \frac{\rho(t)}{t^{n+1}} d t \\
& \approx \sup _{r>0} \varphi_{1}\left(x_{0}, r\right)^{-1} r^{-n}\|f\|_{L_{1}\left(B\left(x_{0}, r\right)\right)} \\
& =\|f\|_{L M_{1, \varphi_{1}}^{\left\{x_{0}\right\}}} \text {. }
\end{aligned}
$$

Corollary 17. Let $1 \leq p<\infty$, the function $\rho$ satisfies the conditions (35), (37), and (42). Let also $\left(\varphi_{1}, \varphi_{2}\right)$ satisfy the conditions

$$
\begin{gathered}
\underset{t<s<\infty}{\operatorname{essinf}} \varphi_{1}(x, s) s^{n / p} \leq C \varphi_{2}\left(x, \frac{t}{2}\right) t^{n / q}, \\
\int_{r}^{\infty}\left(\underset{t<s<\infty}{\operatorname{essinf}} \varphi_{1}(x, s) s^{n / p}\right) \frac{\rho(t)}{t^{n / p+1}} d t \leq C \varphi_{2}(x, r),
\end{gathered}
$$

where $C$ does not depend on $x$ and $r$. Then the operator $I_{\rho}$ is bounded from $M_{p, \varphi_{1}}$ to $M_{q, \varphi_{2}}$ for $p>1$ and from $M_{1, \varphi_{1}}$ to $W M_{q, \varphi_{2}}$ for $p=1$.
In the case $\rho(t)=t^{\alpha}$ from Theorem 16 we get new Spannetype result on generalized local Morrey spaces.

Corollary 18. Let $x_{0} \in \mathbb{R}^{n}, 0<\alpha<n, 1 \leq p<q<\infty$, and $1 / p-1 / q=\alpha / n$. Let also $\left(\varphi_{1}, \varphi_{2}\right)$ satisfy the condition

$$
\int_{r}^{\infty}\left(\underset{t<s<\infty}{\operatorname{essinf}} \varphi_{1}\left(x_{0}, s\right) s^{n / p}\right) \frac{d t}{t^{n / q+1}} d t \leq C \varphi_{2}\left(x_{0}, r\right),
$$

where $C$ does not depend on $r$. Then the operator $I_{\alpha}$ is bounded from $L M_{p, \varphi_{1}}^{\left\{x_{0}\right\}}$ to $L M_{q, \varphi_{2}}^{\left\{x_{0}\right\}}$ for $p>1$ and from $L M_{1, \varphi_{1}}^{\left\{x_{0}\right\}}$ to $W L M_{q, \varphi_{2}}^{\left\{x_{0}\right\}}$ for $p=1$.

Also in the cases $\rho(t)=t^{\alpha}$ and $\varphi(x, t)=t^{(\lambda-n) / p}, 0<$ $\lambda<n$, from Theorem 16 we get local Morrey space variant of Theorem 4 .

Corollary 19. Let $x_{0} \in \mathbb{R}^{n}, 0<\alpha<n, 1<p<n / \alpha$, and $0<\lambda<n-\alpha p$. Moreover, let $1 / p-1 / q=\alpha / n$ and $\lambda / p=\mu / q$. Then, for $p>1$, the operator $I_{\alpha}$ is bounded from $L M_{p, \lambda}^{\left\{x_{0}\right\}}$ to $L M_{q, \lambda}^{\left\{x_{0}\right\}}$ and, for $p=1, I_{\alpha}$ is bounded from $L M_{1, \lambda}^{\left\{x_{0}\right\}}$ to WL $M_{q, \lambda}^{\left\{x_{0}\right\}}$.

\section{Adams-Type Result for the Operator $I_{\rho}$ in $M_{p, \varphi}$}

The following Adams-type result was proved in [31] (see also [12]).

Theorem 20. Let $1 \leq p<\infty, 0<\alpha<n / p$, and $q>p$ and let $\varphi(x, t)$ satisfy the conditions

$$
\begin{gathered}
\sup _{r<t<\infty} \varphi(x, t) \leq C \varphi(x, r), \\
\int_{r}^{\infty} t^{\alpha} \varphi(x, t)^{1 / p} \frac{d t}{t} \leq C r^{-\alpha p /(q-p)},
\end{gathered}
$$

where $C$ does not depend on $x \in \mathbb{R}^{n}$ and $r>0$.

Then the operator $I_{\alpha}$ is bounded from $M_{p, \varphi^{1 / p}}$ to $M_{q, \varphi^{1 / q}}$ for $p>1$ and from $M_{1, \varphi}$ to $W M_{q, \varphi^{1 / q}}$ for $p=1$.

The following Theorem was proved in [32].

Theorem 21. Let $1 \leq p<\infty$ and $\left(\varphi_{1}, \varphi_{2}\right)$ satisfies the condition

$$
\sup _{r<t<\infty} \varphi_{1}(x, t) \leq C \varphi_{2}(x, r)
$$

where $C$ does not depend on $x$ and $r$. Then, for $p>1$, the Hardy-Littlewood maximal operator $M$ is bounded from $M_{p, \varphi_{1}}$ to $M_{p, \varphi_{2}}$ and, for $p=1, M$ is bounded from $M_{1, \varphi_{1}}$ to $W M_{1, \varphi_{2}}$.

The following theorem is a main result of this paper on Adams-type estimate for generalized fractional integral operator $I_{\rho}$. In the case $\rho(t)=t^{\alpha}$ we get Theorem 20 from this theorem. 
Theorem 22. Let $1 \leq p<\infty, q>p$, and $\rho(t)$ satisfy the conditions (37) and (42). Let also $\varphi(x, t)$ satisfy the condition (60) and

$$
\int_{r}^{\infty} \varphi(x, t)^{1 / p} \frac{\rho(t)}{t} d t \leq C \rho(r)^{-p /(q-p)},
$$

where $C$ does not depend on $x \in \mathbb{R}^{n}$ and $r>0$.

Then the operator $I_{\rho}$ is bounded from $M_{p, \varphi^{1 / p}}$ to $M_{q, \varphi^{1 / q}}$ for $p>1$ and from $M_{1, \varphi}$ to $W M_{q, \varphi^{1 / q}}$ for $p=1$.

Proof. Let $x_{0} \in \mathbb{R}^{n}, 1<p<\infty, 0<\alpha<n / p, q>p$, and $f \in M_{p, \varphi^{1 / p}}$. Write $f=f_{1}+f_{2}$, where $B=B(x, r), f_{1}=f \chi_{2 B}$, and $f_{2}=f \chi_{C_{(2 B)}}$. Then we have

$$
I_{\rho} f(x)=I_{\rho} f_{1}(x)+I_{\rho} f_{2}(x) .
$$

For $I_{\rho} f_{1}(y), y \in B(x, r)$, following Hedberg's trick (see, e.g., [33, page 354]), we obtain

$$
\begin{aligned}
\left|I_{\rho} f_{1}(y)\right| & \leq \int_{B(x, 2 r)} \frac{\rho(|y-z|)}{|y-z|^{n}}|f(z)| d z \\
& \approx \sum_{k=-\infty}^{0} \int_{B\left(x, 2^{k+1} r\right) \backslash B\left(x, 2^{k} r\right)} \frac{\rho(|y-z|)}{|y-z|^{n}}|f(z)| d z \\
& \lesssim \sum_{k=-\infty}^{0} \int_{\left(2^{k+1}-1\right) k_{1} r}^{\left(2^{k+1}-1\right) k_{2} r} \frac{\rho(s)}{s^{n+1}} d s \int_{B\left(x, 2^{k+1} r\right)}|f(z)| d z \\
& \approx M f(x) \sum_{k=-\infty}^{0} \int_{\left(2^{k+1}-1\right) k_{1} r}^{\left(2^{k+1}-1\right) k_{2} r} \frac{\rho(s)}{s} d s \\
& =M f(x) \int_{0}^{k_{2} r} \frac{\rho(s)}{s} d s \\
& =M f(x) \tilde{\rho}\left(k_{2} r\right) \\
& \lesssim M f(x) \rho(r) .
\end{aligned}
$$

For $I_{\rho} f_{2}(y), y \in B(x, r)$, from (49) we have

$$
\begin{aligned}
\left|I_{\rho} f_{2}(y)\right| & \lesssim \int_{c_{B(x, 2 r)}} \frac{\rho(|y-z|)}{|y-z|^{n}}|f(z)| d z \\
& \lesssim \int_{2 r}^{\infty}\|f\|_{L_{p}(B(x, t))} \frac{\rho(t)}{t^{n / p+1}} d t .
\end{aligned}
$$

Then from condition (63) and inequality (66) for all $y \in$ $B(x, r)$ we get

$$
\begin{aligned}
\left|I_{\rho} f(y)\right| & \leq \rho(r) M f(x)+\int_{r}^{\infty}\|f\|_{L_{p}(B(x, t))} \frac{\rho(t)}{t^{n / p+1}} d t \\
& \leq \rho(r) M f(x)+\|f\|_{M_{p, q^{1 / p}}} \int_{r}^{\infty} \varphi(x, t)^{1 / p} \frac{\rho(t)}{t} d t \\
& \leq \rho(r) M f(x)+\rho(r)^{-p /(q-p)}\|f\|_{M_{p, q^{1 / p}}} .
\end{aligned}
$$

Hence choosing $\rho(r)=\left(\|f\|_{M_{p, \varphi^{1 / p}}} / M f(x)\right)^{(q-p) / q}$ for all $y \in B(x, r)$, we have

$$
\left|I_{\rho} f(y)\right| \lesssim(M f(x))^{p / q}\|f\|_{M_{p, q^{1 / p}}}^{1-p / q} .
$$

Consequently the statement of the theorem follows in view of the boundedness of the maximal operator $M$ in $M_{p, \varphi^{1 / p}}$ provided by Theorem 21 in virtue of condition (60). If $1<$ $p<q<\infty$, then

$$
\begin{aligned}
& \left\|I_{\rho} f\right\|_{M_{q, \varphi^{1 / q}}} \\
& \quad=\sup _{x \in \mathbb{R}^{n}, t>0} \varphi(x, t)^{-1 / q} t^{-n / q}\left\|I_{\rho} f\right\|_{L_{q}(B(x, t))} \\
& \quad \leq\|f\|_{M_{p, \varphi^{1 / p}}^{1-p / q}} \sup _{x \in \mathbb{R}^{n}, t>0} \varphi(x, t)^{-1 / q} t^{-n / q}\|M f\|_{L_{p}(B(x, t))}^{p / q} \\
& \quad=\|f\|_{L M_{p, \varphi^{1 / p}}}^{1-p / q}\left(\sup _{x \in \mathbb{R}^{n}, t>0} \varphi(x, t)^{-1 / p} t^{-n / p}\|M f\|_{L_{p}(B(x, t))}\right)^{p / q} \\
& \quad=\|f\|_{M_{p, \varphi^{1 / p}}^{1-p / q}}\|M f\|_{M_{p, \varphi^{1 / p}}^{p / q}} \\
& \quad \leq\|f\|_{M_{p, \varphi^{1 / p}},}
\end{aligned}
$$

and if $1<q<\infty$, then

$$
\begin{aligned}
& \left\|I_{\rho} f\right\|_{W M_{q, \varphi^{1} / q}} \\
& \quad=\sup _{x \in \mathbb{R}^{n}, t>0} \varphi(x, t)^{-1 / q} t^{-n / q}\left\|I_{\rho} f\right\|_{W L_{q}(B(x, t))} \\
& \quad \leq\|f\|_{M_{1, \varphi}}^{1-1 / q} \sup _{x \in \mathbb{R}^{n}, t>0} \varphi(x, t)^{-1 / q} t^{-n / q}\|M f\|_{W L_{1}(B(x, t))}^{1 / q} \\
& \quad=\|f\|_{M_{1, \varphi}}^{1-1 / q}\left(\sup _{x \in \mathbb{R}^{n}, t>0} \varphi(x, t)^{-1} t^{-n}\|M f\|_{W L_{1}(B(x, t))}\right)^{1 / q} \\
& \quad=\|f\|_{M_{1, \varphi}}^{1-1 / q}\|M f\|_{W M_{1, \varphi}}^{1 / q} \\
& \quad \leq\|f\|_{M_{1, \varphi}} .
\end{aligned}
$$

Thus the proof of the theorem is completed.

\section{Conflict of Interests}

The authors declare that there is no conflict of interests regarding the publication of this paper.

\section{Acknowledgments}

The authors would like to express their gratitude to the referees for their very valuable comments and suggestions. V. S. Guliyev was partially supported by the grant of Science Development Foundation under the President of the Republic of Azerbaijan, Grant EIF-2013-9(15)-46/10/1, and by the 
grant of Ahi Evran University Scientific Research Projects (PYO.FEN.4003-2.13.007).

\section{References}

[1] C. B. Morrey, "On the solutions of quasi-linear elliptic partial differential equations," Transactions of the American Mathematical Society, vol. 43, no. 1, pp. 126-166, 1938.

[2] A. L. Mazzucato, "Besov-Morrey spaces: function space theory and applications to non-linear PDE," Transactions of the American Mathematical Society, vol. 355, no. 4, pp. 1297-1364, 2003.

[3] M. E. Taylor, "Analysis on Morrey spaces and applications to Navier-Stokes and other evolution equations," Communications in Partial Differential Equations, vol. 17, no. 9-10, pp. 1407-1456, 1992.

[4] A. D. Gadjiev, "On generalized potential-type integral operators," Functiones et Approximatio Commentarii Mathematici, vol. 25, pp. 37-44, 1997.

[5] V. S. Guliyev and R. Ch. Mustafaev, "On generalized fractional integrals," Transactions of Academy of Sciences of Azerbaijan. Series of Physical-Technical and Mathematical Sciences, vol. 21, no. 4, pp. 63-71, 237, 2001.

[6] E. Nakai, "On generalized fractional integrals," Taiwanese Journal of Mathematics, vol. 5, no. 3, pp. 587-602, 2001.

[7] A. Eridani, "On the boundedness of a generalized fractional integral on generalized Morrey spaces," Tamkang Journal of Mathematics, vol. 33, no. 4, pp. 335-340, 2002.

[8] H. Gunawan, "A note on the generalized fractional integral operators," Journal of the Indonesian Mathematical Society, vol. 9, no. 1, pp. 39-43, 2003.

[9] A. Eridani, H. Gunawan, and E. Nakai, "On generalized fractional integral operators," Scientiae Mathematicae Japonicae, vol. 60, no. 3, pp. 539-550, 2004.

[10] E. Nakai, "On generalized fractional integrals on the weak Orlicz spaces, $B M O^{\varphi}$, the Morrey spaces and the Campanato spaces," in Function Spaces, Interpolation Theory and Related Topics, pp. 389-401, Walter De Gruyter, Berlin, Germany, 2000.

[11] A. Eridani, H. Gunawan, E. Nakai, and Y. Sawano, "Characterizations for the generalized fractional integral operators on Morrey spaces," Mathematical Inequalities \& Applications, vol. 17, no. 2, pp. 761-777, 2014.

[12] V. S. Guliyev, "Boundedness of the maximal, potential and singular operators in the generalized Morrey spaces," Journal of Inequalities and Applications, vol. 2009, Article ID 503948, 20 pages, 2009.

[13] V. S. Guliyev, Integral operators on function spaces on the homogeneous groups and on domains in Rn [Ph.D. dissertation], Steklov Institute of Mathematics, Moscow, Russia, 1994, (Russian).

[14] V. S. Guliyev, Function Spaces, Integral Operators and Two Weighted Inequalities on Homogeneous Groups. Some Applications, Casioglu, Baku, Azerbaijan, 1999, (Russian).

[15] N. Wiener, "Generalized harmonic analysis," Acta Mathematica, vol. 55, no. 1, pp. 117-258, 1930.

[16] N. Wiener, "Tauberian theorems," Annals of Mathematics, vol. 33, no. 1, pp. 1-100, 1932.

[17] A. Beurling, "Construction and analysis of some convolution algebras," Annales de l'Institut Fourier, vol. 14, pp. 1-32, 1964.
[18] H. Feichtinger, "An elementary approach to Wieners third Tauberian the-orem on Euclidean n-space," in Harmonic Analysis, Symmetric Spaces and Probability Theory, Cortona/Italy 1984, Symp. Math., pp. 267-301, Academic Press, London, UK, 1987.

[19] J. Alvárez, J. Lakey, and M. Guzmán-Partida, "Spaces of bounded $\lambda$-central mean oscillation, Morrey spaces, and $\lambda$ central Carleson measures," Collectanea Mathematica, vol. 51, no. 1, pp. 1-47, 2000.

[20] J. Peetre, "On the theory of $\mathscr{L}_{p, \lambda}$," Journal of Functional Analysis, vol. 4, no. 1, pp. 71-87, 1969.

[21] D. R. Adams, "A note on Riesz potentials," Duke Mathematical Journal, vol. 42, no. 4, pp. 765-778, 1975.

[22] V. S. Guliyev and Y. Sawano, "Linear and sublinear operators on generalized Morrey spaces with non-doubling measures," Publicationes Mathematicae Debrecen, vol. 83, no. 3, pp. 303327, 2013.

[23] E. Nakai, "Hardy-Littlewood maximal operator, singular integral operators and the Riesz potentials on generalized Morrey spaces," Mathematische Nachrichten, vol. 166, pp. 95-103, 1994.

[24] N. Samko, "Maximal, potential and singular operators in vanishing generalized Morrey spaces," Journal of Global Optimization, vol. 57, no. 4, pp. 1385-1399, 2013.

[25] L. G. Softova, "Singular integrals and commutators in generalized Morrey spaces," Acta Mathematica Sinica-English Series, vol. 22, no. 3, pp. 757-766, 2006.

[26] T. Mizuhara, "Boundedness of some classical operators on generalized Morrey spaces," in ICM-90 Satellite Conference Proceedings: Harmonic Analysis, pp. 183-189, Springer, Sendai, Japan, 1991.

[27] V. S. Guliyev, S. S. Aliyev, T. Karaman, and P. S. Shukurov, "Boundedness of sublinear operators and commutators on generalized Morrey spaces," Integral Equations and Operator Theory, vol. 71, no. 3, pp. 327-355, 2011.

[28] V. S. Guliyev, "Generalized local Morrey spaces and fractional integral operators with rough kernel," Journal of Mathematical Sciences, vol. 193, no. 2, pp. 211-227, 2013.

[29] V. S. Guliyev, "Generalized weighted Morrey spaces and higher order commutators of sublinear operators," Eurasian Mathematical Journal, vol. 3, no. 3, pp. 33-61, 2012.

[30] Y. Sawano, S. Sugano, and H. Tanaka, "Orlicz-Morrey spaces and fractional operators," Potential Analysis, vol. 36, no. 4, pp. 517-556, 2012.

[31] V. S. Guliyev and P. S. Shukurov, "On the boundedness of the fractional maximal operator, Riesz potential and their commutators in generalized Morrey spaces," in Advances in Harmonic Analysis and Operator Theory, vol. 229 of Operator Theory: Advances and Applications, pp. 175-199, Birkhäauser, 2013.

[32] A. Akbulut, V. Guliyev, and R. Mustafayev, "On the boundedness of the maximal operator and singular integral operators in generalized Morrey spaces," Mathematica Bohemica, vol. 137, no. 1, pp. 27-43, 2012.

[33] E. M. Stein, Harmonic Analysis: Real Variable Methods, Orthogonality and Oscillatory Integrals, Princeton University Press, Princeton, NJ, USA, 1993. 


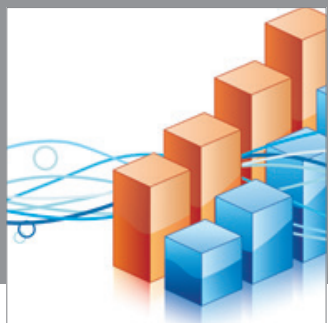

Advances in

Operations Research

mansans

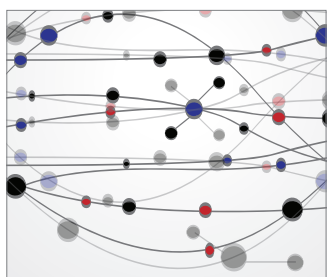

The Scientific World Journal
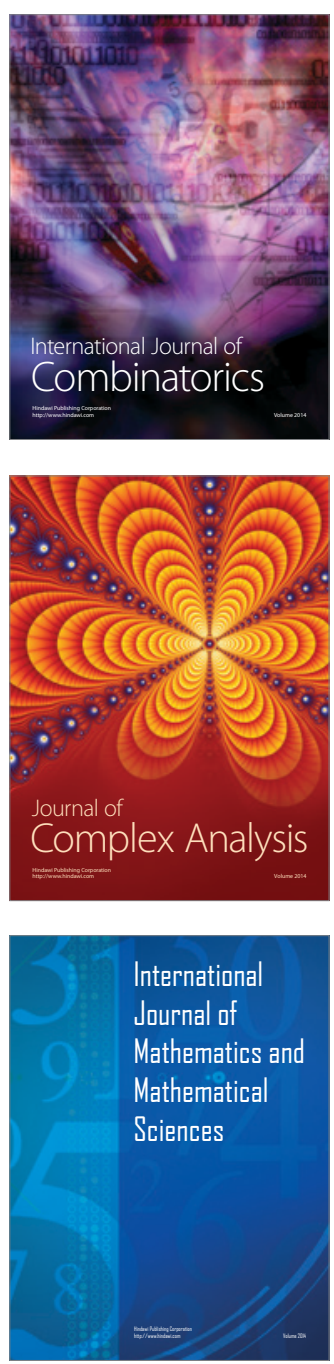
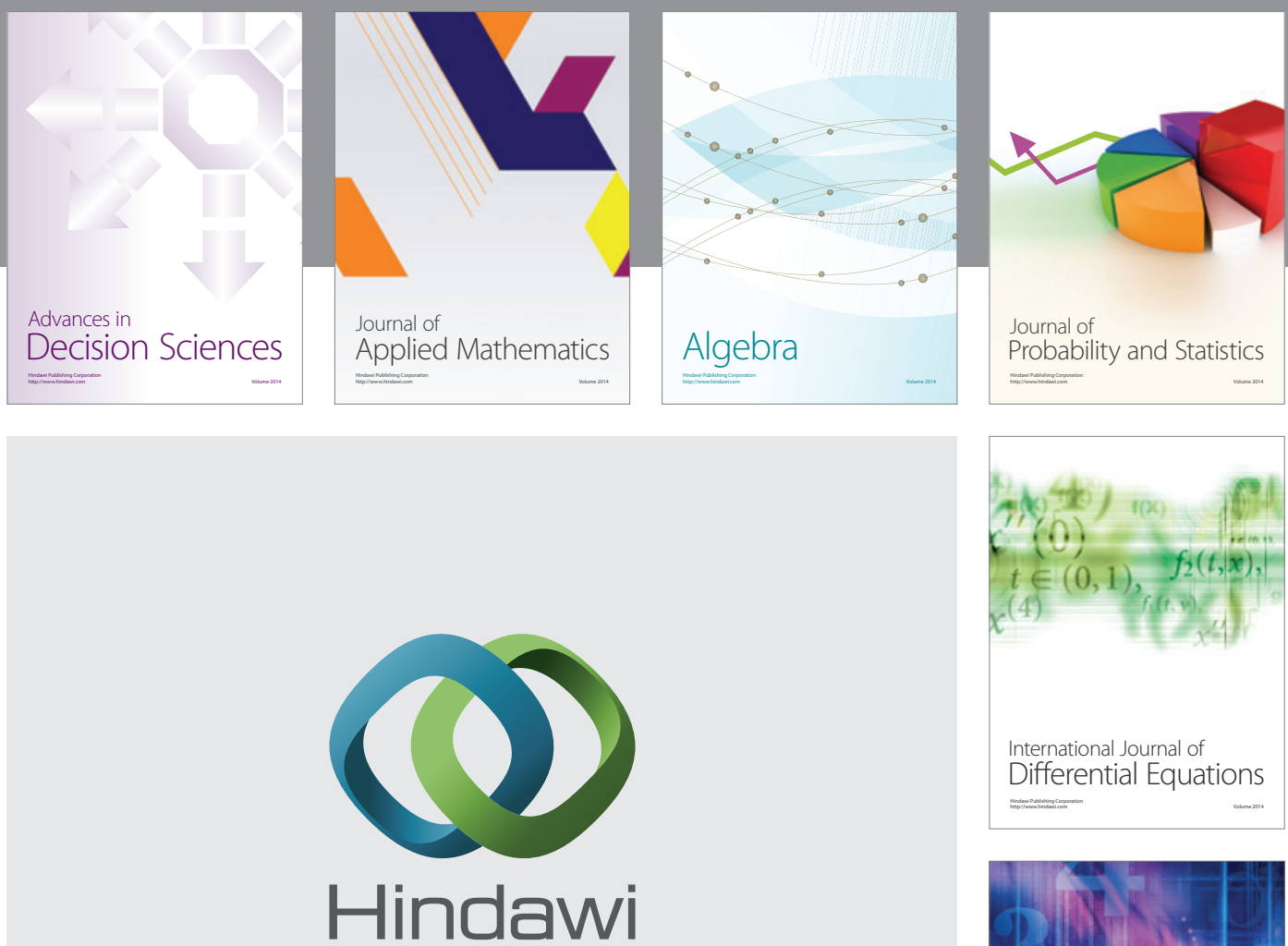

Submit your manuscripts at http://www.hindawi.com
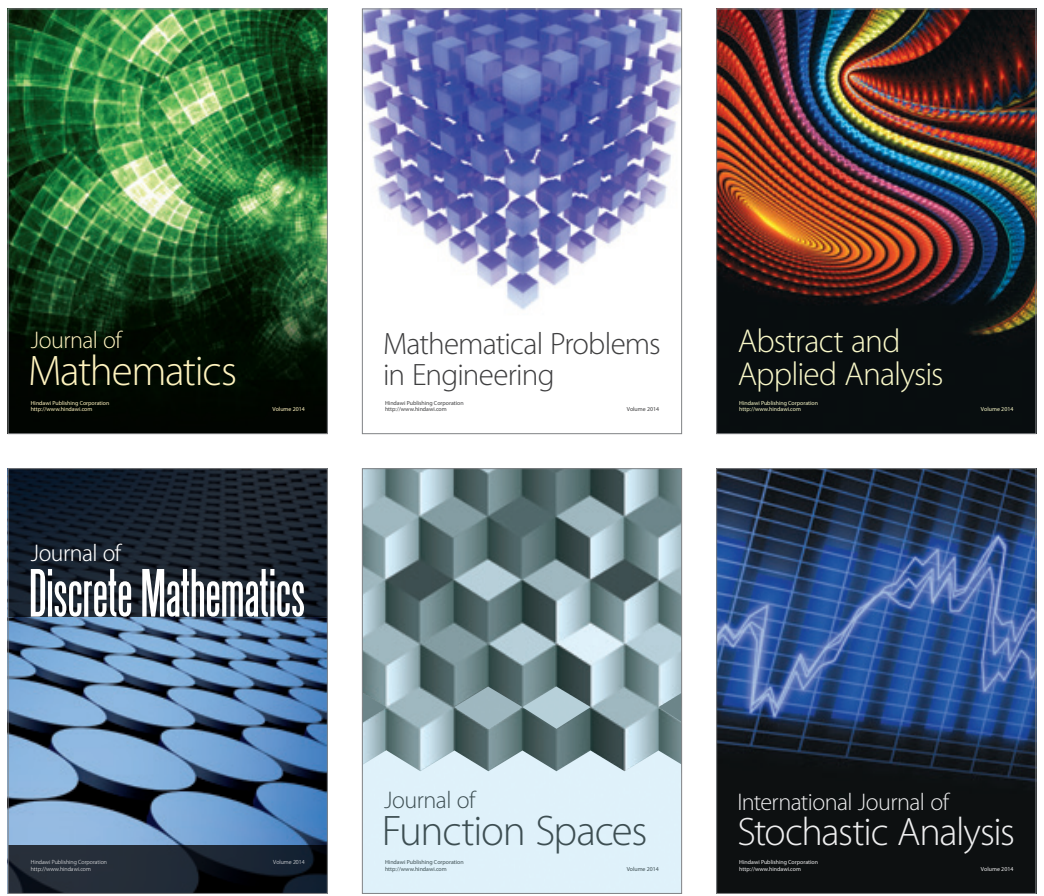

Journal of

Function Spaces

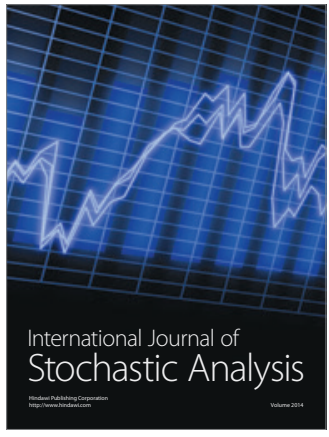

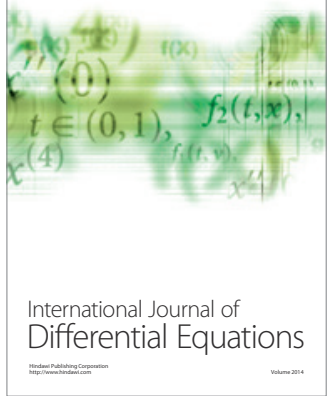
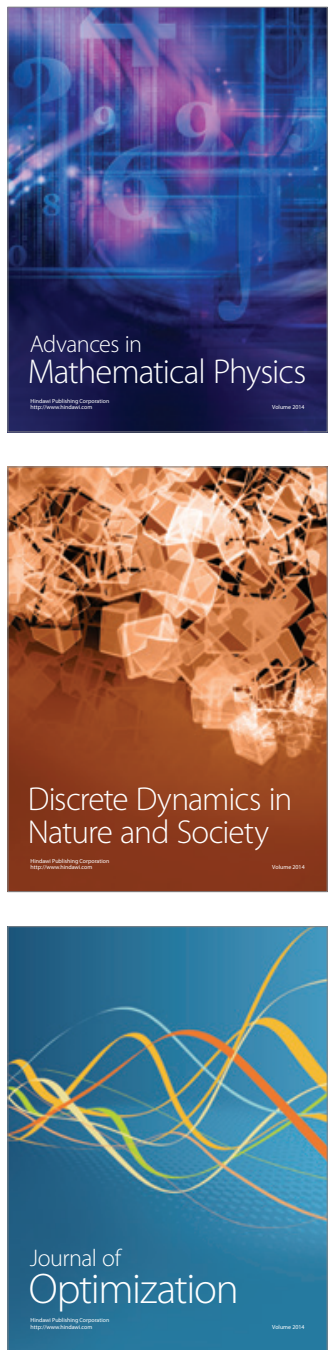